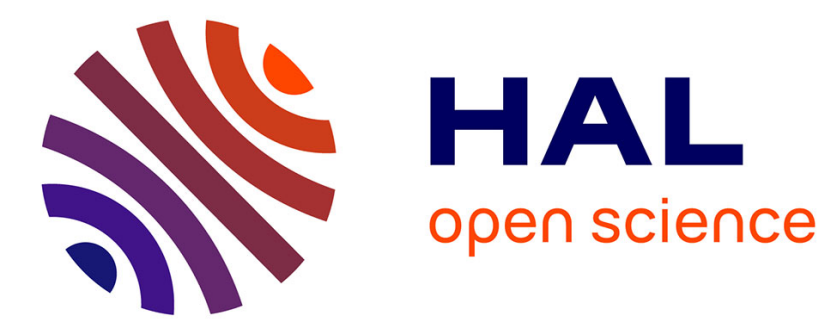

\title{
What can we learn from the energy dependence of strangeness production at RHIC?
}

J. Speltz

\section{To cite this version:}

J. Speltz. What can we learn from the energy dependence of strangeness production at RHIC?. Hot Quarks 2006: Workshop for Young Scientists on the Physics of Ultrarelativistic Nucleus-Nucleus Collisions, May 2006, Villasimius, Italy. pp.103-108, 10.1140/epjc/s10052-006-0104-7 . in2p3-00127908

\section{HAL Id: in2p3-00127908 https://hal.in2p3.fr/in2p3-00127908}

Submitted on 20 Feb 2007

HAL is a multi-disciplinary open access archive for the deposit and dissemination of scientific research documents, whether they are published or not. The documents may come from teaching and research institutions in France or abroad, or from public or private research centers.
L'archive ouverte pluridisciplinaire HAL, est destinée au dépôt et à la diffusion de documents scientifiques de niveau recherche, publiés ou non, émanant des établissements d'enseignement et de recherche français ou étrangers, des laboratoires publics ou privés. 


\title{
What can we learn from the energy dependence of strangeness production at RHIC?
}

\author{
Jeff Speltz (for the STAR Collaboration) \\ Institut Pluridisciplinaire Hubert Curien, 23 rue du Loess, BP 28, Strasbourg, France
}

Received: date / Revised version: date

\begin{abstract}
Measurements of strange and multi-strange particles with the STAR detector at center of mass energies per nucleon pair $\left(\sqrt{s_{N N}}=\right)$ of $62.4 \mathrm{GeV}$ and $200 \mathrm{GeV}$ in ultra-relativistic $\mathrm{Au}+\mathrm{Au}$ collisions at RHIC are presented. The results are discussed in order to get insight into chemical and dynamical properties of the created medium. The former are obtained by comparison of transverse momentum $\left(p_{T}\right)$ integrated observables such as yields and particle ratios to statistical models, while the latter use $p_{T}$ dependent quantities such as single particle spectra and elliptic flow measurements to challenge hydrodynamical model calculations and parameterizations. The discussion is orientated towards the energy dependence of these properties by confronting the results at the different RHIC energies but also with the lower SPS energies.
\end{abstract}

\section{Introduction}

Strangeness observables have been suggested, since the beginning of the study of relativistic heavy ion collisions, to yield possible effects of the creation of a quark gluon plasma [1]. These observables are supposed to give valuable insight into the properties of the created medium, as all strange valence quarks in the final state originate from the collision and are not present in the incoming nuclei. The study of these features in terms of chemical and kinetic freeze-out conditions by comparing the data to predictions of models may then reveal different aspects and characteristics of the fireball they arise from. The examination of the energy dependence of these properties, especially with the results at the intermediate energy of 62.4 $\mathrm{GeV}$ that stands between the top RHIC and the SPS energies, gives the possibility to access a more global picture of the characteristics of the medium, such as a possible onset of the formation of a QGP.

The presented STAR [2] data are from $\mathrm{Au}+\mathrm{Au}$ collisions delivered by RHIC and were measured with its main tracking device, the Time Projection Chamber (TPC) [3]. The $200 \mathrm{GeV}$ results are from the RUN II [4] and the 62.4 $\mathrm{GeV}$ results come from the RUN IV $[5,6]$. The results are for mid-rapidity $(|y|<1)$ if not otherwise stated.

\section{Chemical Properties}

The relative aboundance of the different particle species and consequently the chemical composition of the medium is frozen at chemical freeze-out which marks the end of inelastic interactions among the particles. The temperature, $T_{c h}$, that characterizes this freeze-out can be accessed by statistical models [7-11]. These models compute the production rates of the different particles by using a limited set of parameters and assume a statistically equilibrated matter. Hence one can consider that these parameters allow the caracterization of the chemical properties of the system. Besides $T_{c h}$, two chemical potentials are used, one for the light quarks $\mu_{q}(q=u, d$; baryon chemical potential $\left.\mu_{B}=3 \mu_{q}\right)$ and one for the strange quarks $\mu_{S}$. They both regulate anti-particle relative to particle production. Additionally a strangeness phase-space occupancy factor, $\gamma_{S}$, that caracterizes the strangeness saturation and indicates whether strangeness production has reached its equilibrium level or not. For a given collision energy and system, these parameters are obtained by adjusting measured particle ratios.

In Fig.1(top panel) particle ratios obtained from 62.4 $\mathrm{GeV}$ central $\mathrm{Au}+\mathrm{Au}$ data (circles) are shown with results from statistical model calculations obtained by adjusting data ratios involving $\pi^{ \pm}, K^{ \pm}, p$ and $\bar{p}, \Lambda$ and $\Xi$ (lines) with a $\chi^{2} / n d f=2.3 / 5$. The results on the $\Omega / \pi$ and $\bar{\Omega} / \Omega$ ratios are then a pure prediction from the statistical model. The difference between data and model prediction is evaluated by the number of standard deviations as shown in the bottom panel of Fig.1. A comparable figure, exposing the good agreement between data and statistical model calculations at $200 \mathrm{GeV}$, can be found elsewhere [12].

Fig. 2 shows the dependence of $T_{c h}$ and $\gamma_{S}$ on the mean number of participant nucleons, $\left\langle N_{\text {part }}\right\rangle$, determined by a Glauber model calculation [13]. The closed circles are for $62.4 \mathrm{GeV}$ and the open cercles are for $200 \mathrm{GeV}$. The results at both energies were obtained with the same model [11]. The temperature is constant over all centralities at $\sim 160 \mathrm{MeV}$, close to the critical temperature of $T_{c} \sim 170 \mathrm{MeV}$ 


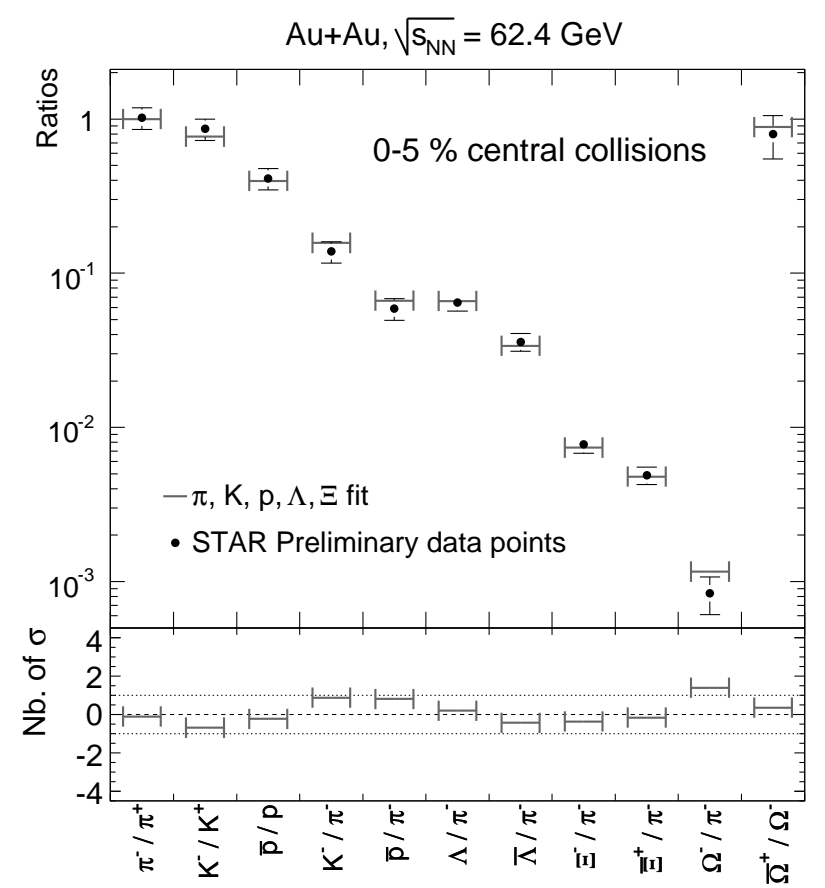

Fig. 1. Top panel: "Stable" particle ratios obtained by measurements for most central collisions at $\sqrt{s_{N N}}=62.4 \mathrm{GeV}$ (points) superposed with statistical model calculations using [11] (lines). The experimental data have statistical and systematical errors added quadratically. Bottom panel:Number of standard deviations $(\sigma)$ between data and model calculations.

obtained from lattice QCD calculations [14]. In contrast, $\gamma_{S}$ monotonously rises from a around 0.7 for peripheral collisions to saturate at a value close to unity for the most central collisions. This indicates the presence of a state of matter where strangeness production is close to saturation and may thus reveal the achievement of a global chemical equilibrium for strangeness. The results for 200 $\mathrm{GeV}$ shown on the same figure as open circles display the same behaviour and magnitude within error bars as the 62.4 GeV data, while the SPS data at the lower energy of $17.3 \mathrm{GeV}$ may give a slightly lower value for $\gamma_{S}[9]$, with a comparable centrality dependence.

The baryon chemical potential obtained at $62.4 \mathrm{GeV}$ $\left(\mu_{B}=87 \pm 13 \mathrm{MeV}\right)$ is higher as at $200 \mathrm{GeV}\left(\mu_{B}=24 \pm 4\right.$ $\mathrm{MeV}$ ) but stands where expected to be by interpolation between SPS and RHIC results [15]. This variation in $\mu_{B}$ in statistical models results directly from the the antibaryon to baryon ratios in data. In Fig. 3 the evolution of this ratio for $\Lambda, \Xi$ and $\Omega$ is represented as a function of $\sqrt{s_{N N}}$. The smooth rise in these ratios and the approach to unity for top RHIC energy translates the transition from a transport dominated regime to a dominance of quark anti-quark pair production. The latter leads to a baryon-free environment equivalent to lower baryon chemical potential.

The astonishing success of statistical models over a large range of energies, especially also for $\mathrm{p}+\mathrm{p}$ and $\mathrm{e}^{-}+$ $\mathrm{e}^{+}$collisions [16] requires a word of caution in the inter-

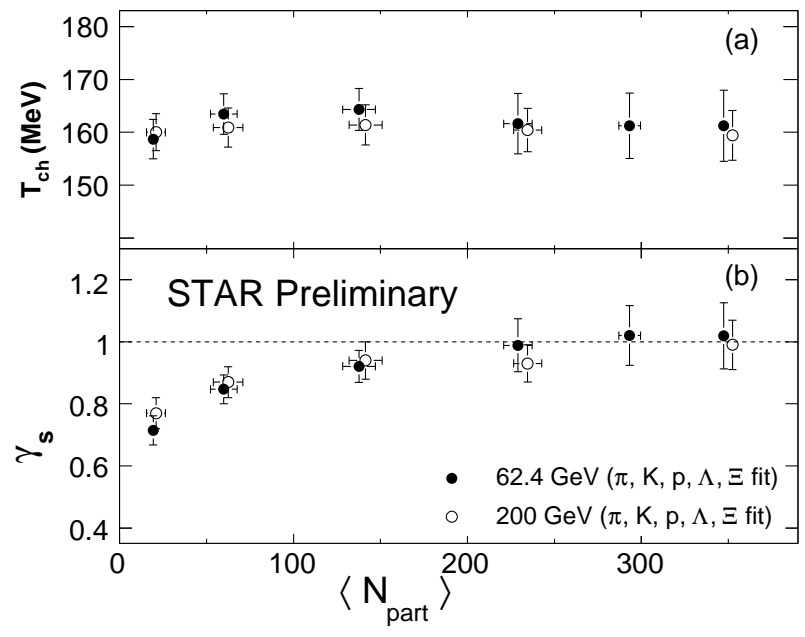

Fig. 2. Centrality dependence $\left(\left\langle N_{\text {part }}\right\rangle\right)$ of (a) chemical freezeout temperature $T_{c h}$ and (b) strangeness saturation factor $\gamma_{S}$ for $62.4 \mathrm{GeV}$ (closed circles) and $200 \mathrm{GeV}$ (open circles). Statistical and systematic errors are added quadratically.

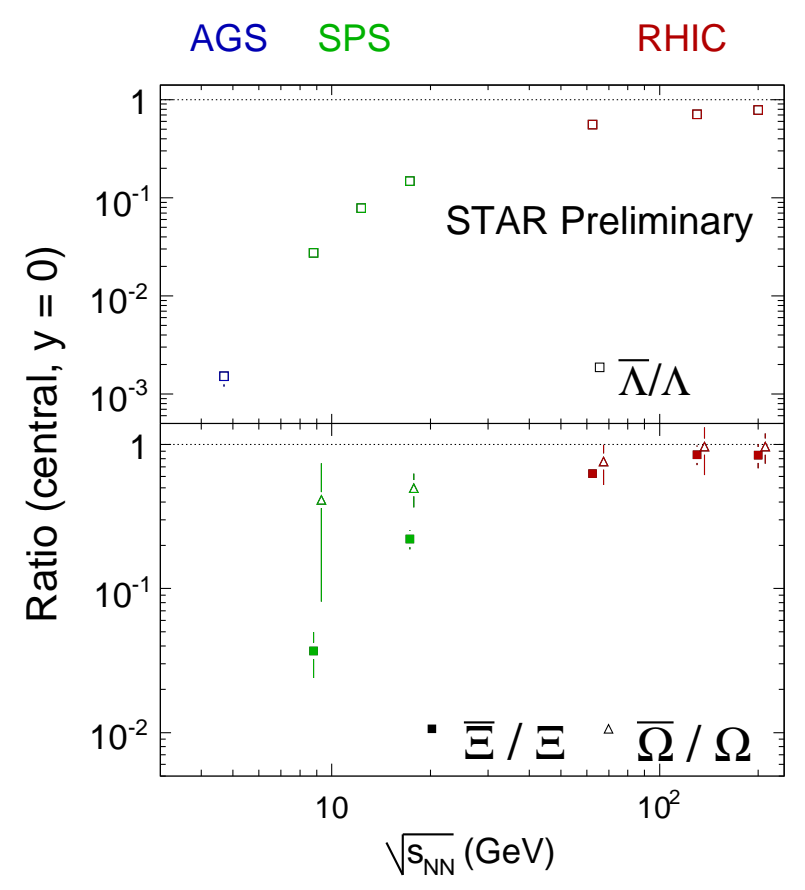

Fig. 3. Energy dependence $\left(\sqrt{s_{N N}}\right)$ of $\bar{\Lambda} / \Lambda, \bar{\Xi} / \Xi$ and $\bar{\Omega} / \Omega$ ratios for central nucleus-nucleus collisions at mid-rapidity [17].

pretation with respect to a chemical equilibration of the system. It is possible that it only looks as if the system follows statistical laws because the experimental ratios result from averaging over a large number of events. The abundances from a single event may then not necessarily be reproducable by models assuming chemical equilibrium. 


\section{Dynamical Properties}

Dynamical properties of the system created in relativistic heavy-ion collisions can be accessed by $p_{T}$ dependent observables, such as particle spectra and differential elliptic flow [18]. The comparison of data with models may help getting information on these properties, such as the temperature $T_{d e c}\left(T_{k i n}\right)$ of the kinetic freeze-out when elastic interactions end and the magnitude of collective motion during the expansion. In turn, these should give insight into the validation or not of the hypothesis of local thermalization and even the creation of a de-confined phase of quarks and gluons which should be described by a dedicated equation of state.

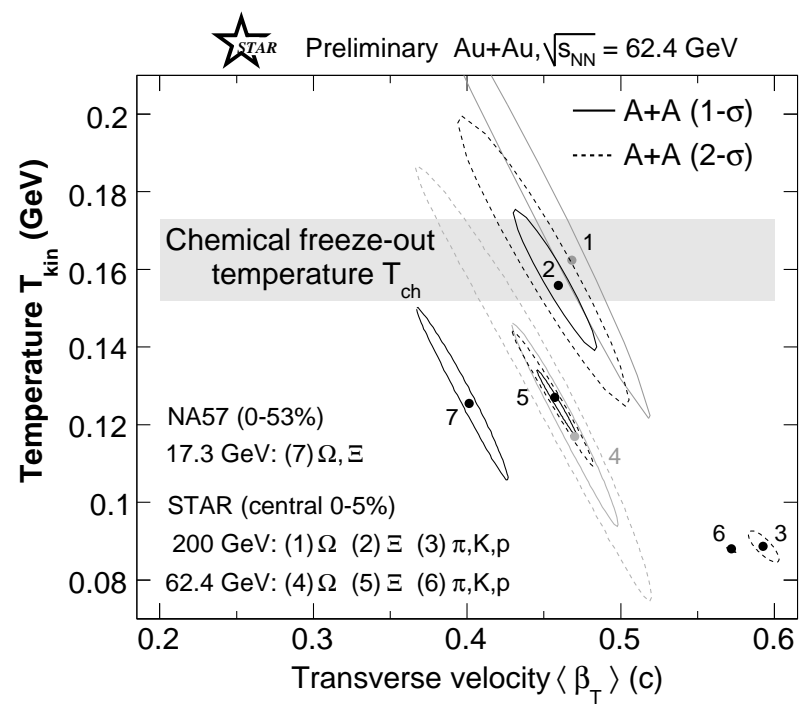

Fig. 4. One and two $\sigma$ contours in $T_{k i n}-\left\langle\beta_{T}\right\rangle$ space obtained by blast-wave fits on the measured spectra from most central collisions at different energies and for different particle species. The NA57 contour is from [21].

At first, we extract dynamical parameters with the hydrodynamically inspired, so-called Blast-Wave(B-W) parameterization $[19,20]$, by performing a direct fit on the spectra. The two free parameters in the fit, $T_{k i n}$ and $\left\langle\beta_{T}\right\rangle$ are interpreted as the kinetic freeze-out temperature and the mean transverse flow velocity, respectively. Fig. 4 shows the confidence-level contours in $T_{k i n}-\left\langle\beta_{T}\right\rangle$ space obtained by blast-wave fits on the spectra of different particle species at different energies. All experimental spectra are inclusive, meaning that they are not corrected for contributions from resonances and weak feed-down. The $62.4 \mathrm{GeV}$ and $200 \mathrm{GeV}$ contours reveal a clear discrepancy between the transverse flow of the light particles $\left(\pi^{ \pm}, K^{ \pm}, p\right.$ and $\left.\bar{p}\right)$ and the multi-strange baryons $(\Xi$ and $\Omega$ ). For the light particles, a decrease in radial flow is observed for the lower energy. Such a decrease for multi-strange baryons is not easy to identify between the $200 \mathrm{GeV}$ and the $62.4 \mathrm{GeV}$ data points due to the uncertainties. But it may be observed when comparing to the NA57 results [21] at 17.3 $\mathrm{GeV}$. A caveat is that the NA57 contour is for a much larger centrality range which includes peripheral collisions that may lead to less radial flow. A disagreement may also be seen on $T_{k i n}$. The values of $T_{k i n}$ seem to be higher for the multi-strange baryons than those obtained by a combined fit for the light particles. Given the large errors, this seems to be especially the case at $200 \mathrm{GeV}$ and is not as obvious at $62.4 \mathrm{GeV}$.

For a more accurate comparison with ideal hydrodynamics, we use a model by Kolb et al. that numerically solves the hydrodynamical equations. Computations have been published for the energy of $200 \mathrm{GeV}$ [22] and as it is publicly available on the web [23], we used it to get predictions for the energy of $62.4 \mathrm{GeV}$. The applied equation of state is the same as that at $200 \mathrm{GeV}$ and includes a first order phase transition from a QGP to a hadron gas at $T_{c}=165 \mathrm{MeV}$. The model assumes partial chemical equilibrium in order to reproduce the aboundances of the different particle species. Chemical equilibrium is maintained in the QGP phase and a chemical freeze-out occurs at $T_{c h}=164 \mathrm{MeV}$. The initial entropy and baryon densities have been adjusted to reproduce the measured multiplicity and $p / \pi$ and $\bar{p} / p$ ratios at $62.4 \mathrm{GeV}$. This model uses an additional parameter, noted $\alpha$, that parameterizes a possible initial transverse boost that may have been developed prior to thermalization. The time from which the thermalization hypothesis is valid is parameterized by $\tau_{0}$. Its value at $62.4 \mathrm{GeV}$ has been taken to be the same as at $200 \mathrm{GeV}[22]$, namely $\tau_{0}=0.6 \mathrm{fm} / \mathrm{c}$.

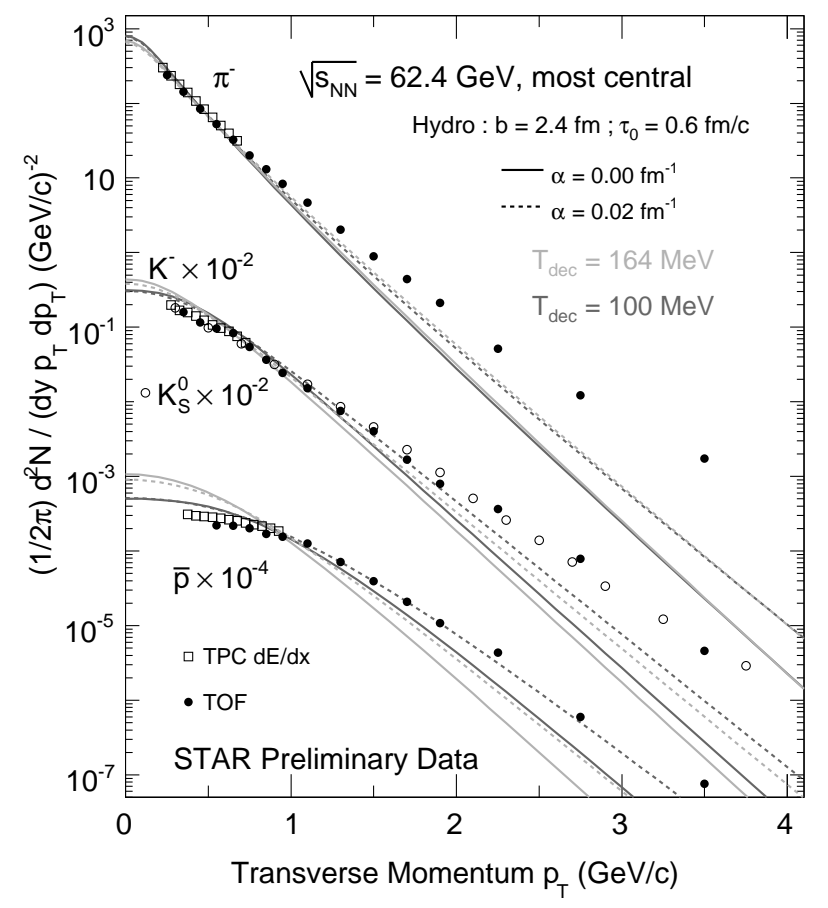

Fig. 5. Measured $\pi^{-}, K^{-}, K_{S}^{0}$ and $\bar{p}$ spectra (points) for most central $\mathrm{Au}+\mathrm{Au}$ collisions at $62.4 \mathrm{GeV}$ superposed with hydrodynamical calculations (lines) for different freeze-out temperatures $\left(T_{k i n}\right)$ and $\alpha$ parameters obtained by using $[22,23]$. 


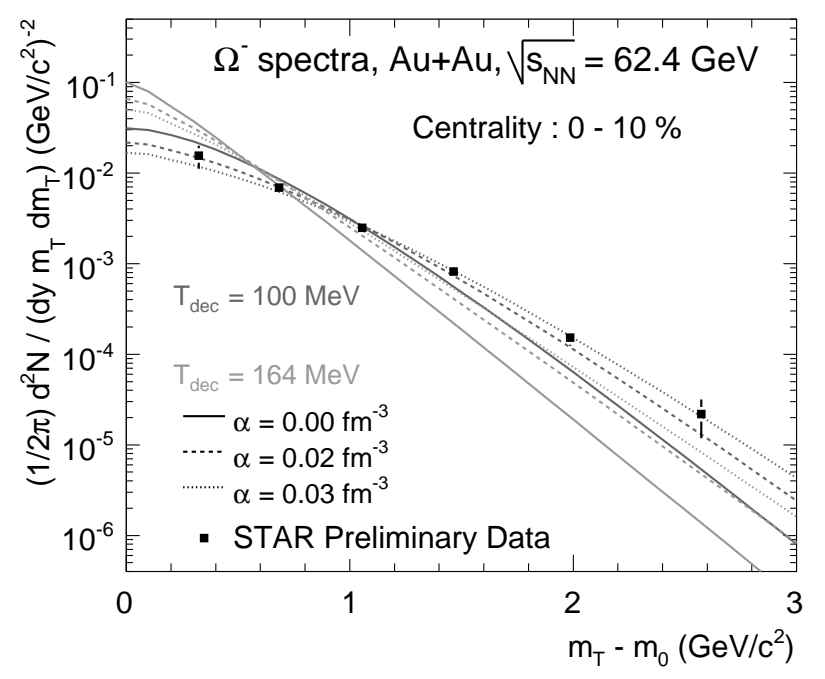

Fig. 6. Measured transverse mass $\left(m_{T}=\sqrt{p_{T}^{2}+m_{0}^{2}}\right) \Omega$ spectra (points) from most central $\mathrm{Au}+\mathrm{Au}$ collisions at $62.4 \mathrm{GeV}$ superposed with various hydrodynamical calculations (lines) for different $T_{d e c}$ and $\alpha$ obtained by using [22,23].

Fig.5 shows the spectra for light particles $\left(\pi^{-}, K^{-}\right.$, $K_{S}^{0}$ and $\bar{p}$ ) for most central $\mathrm{Au}+\mathrm{Au}$ collisions at $62.4 \mathrm{GeV}$ superposed with hydrodynamical calculations using the aforementionned adapted model. The agreement between data and model holds for a limited domain in transverse momentum that might be smaller as was observed at 200 $\mathrm{GeV}[22]$. The best agreement at $62.4 \mathrm{GeV}$, as has been at $200 \mathrm{GeV}$, is obtained with a freeze-out temperature of $T_{k i n}=100 \mathrm{MeV}$ and a small but finite value for the $\alpha$ parameter. The agreement between data and hydrodynamical calculations is supposed to hold only at low- $p_{T}(<1.5-$ $2.0 \mathrm{GeV} / \mathrm{c}$ ), as particles with higher $p_{T}$ undergo less rescattering and may therefore verify less the thermalization hypothesis of ideal hydrodynamical calculations. With the current preliminary data, it also looks as if the agreement would hold for higher $p_{T}$ for the heavier particles. This appears to be true also for the $\Omega$, the particle with the highest strangeness content, where the agreement seems to hold for the entire measured spectra as Fig.6 illustrates. The non-chemical equilibrium part of the evolution does not include a strange chemical potential. This may have significant influence on the strange particle multiplicity. Therefore we only discuss the shape of the spectra and not its normalization, that has been sightly adjusted to reproduce the data. A similar observation regarding the agreement between data and hydrodynamical calculations on the $\Omega$ has already been made at $200 \mathrm{GeV}$ [22]. At both energies, it seems that a common freeze-out temperature of $\sim 100 \mathrm{MeV}$ and a non-vanishing $\alpha$ parameter, the same as for the light particles, best reproduce the $\Omega$ data within the framework of these hydrodynamical model calculations. A higher thermal freeze-out temperature, just after hadronisation $\left(T_{\text {dec }}=164 \mathrm{MeV}\right)$, does not develop sufficient radial flow to reproduce the data.

The results regarding the thermal freeze-out temperature from these hydrodynamical calculations seem incom- patible with the observations from the Blast-Wave fits. This apparent discrepancy may have different explanations. First of all, the error bars on the Blast-Wave contours (Fig.4) are still quite large for the multi-strange baryons. The shown contours do not include systematic error induced by the variation of an additional parameter in the Blast-Wave, the velocity profile, which affects particularly the values of the temperature parameter. Additionally the fit-range used for the fit on the different particles is not the same, as the measured spectra do not have the same $p_{T}$ coverage, especially at very low- $p_{T}$ $(<0.7 \mathrm{GeV} / \mathrm{c})$. Further investigations included a direct comparison between the hydrodynamical $\left(T_{d e c}\right)$ and the Blast-Wave $\left(T_{k i n}\right)$ temperature. This was done by performing a Blast-Wave fit on the spectra from the hydrodynamical model with a given temperature. The fit gives a good agreement with the spectra, however the values of the parameters obtained by the Blast-Wave fit are systematically lower than the hydrodynamical temperature with a smaller difference for lower $T_{\text {dec }}(3-10 \mathrm{MeV}$ at $\left.T_{\text {dec }} \sim 100 \mathrm{MeV}\right)$ than at higher $T_{\text {dec }}(15-30 \mathrm{MeV}$ at $\left.T_{\text {dec }} \sim 164 \mathrm{MeV}\right)$. This raises the question whether $T_{d e c}$ (hydro) and $T_{k i n}(\mathrm{~B}-\mathrm{W})$ are both meaningful physical quantities. While the Blast-Wave parameterization needs many simplyfing assumptions, for instance on the velocity profile to obtain a single formula with three parameters, the hydrodynamical model numerically resolves the differential equations for the statistical description of particles. This constitutes a natural and obvious difference between the two approaches.

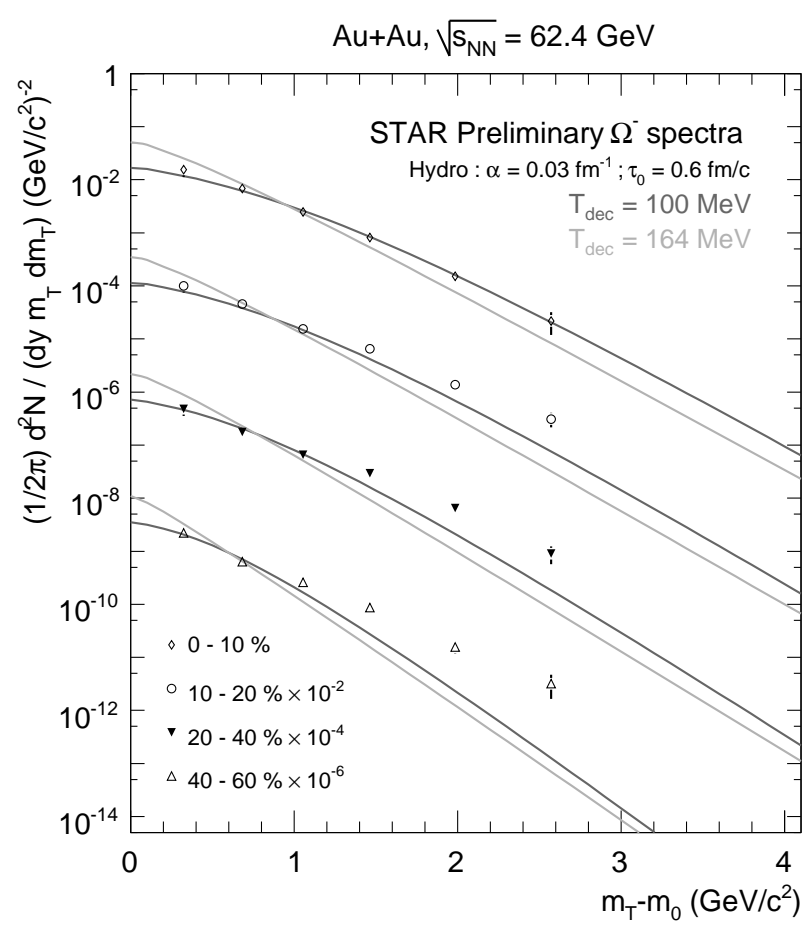

Fig. 7. Centrality dependence of $\Omega$ spectra in $\mathrm{Au}+\mathrm{Au}$ collisions at $62.4 \mathrm{GeV}$. Superposed with data (points) are hydrodynamical calculations (lines) obtained by using [22,23]. 
The $62.4 \mathrm{GeV}$ high statistics data also allows for the study of the centrality dependence of the $\Omega$ spectra shown in Fig.7. The hydrodynamical calculations for the different centrality intervals only differ by the impact parameter, $b$, used in the calculations. All the other parameters are identical to those used for the most central collisions. Only the curves that give the best agreement for the most central collisions (Fig.6; $\alpha=0.03 \mathrm{fm}^{-1}$ ) are shown for two different temperatures. The spectra from hydrodynamical calculations deviate more and more from the data when the collisions become more peripheral. This deviation is expected as for more peripheral collisions, the system size gets smaller so that the thermalization hypothesis is less valid. The centrality dependence may then help quantif the conditions for which the hypothesis of thermal equilibrium is applicable.

Finally, differential elliptic flow behaviour is investigated. Elliptic flow originates from the spatial asymmetry, caused by the almond shape of the collision overlap zone. The interactions among the constitants generate a pressure gradiant which transforms this spatial asymmetry into an asymmetry in momentum space. Hydrodynamical calculations have been shown to qualitatively reproduce the data at $200 \mathrm{GeV}$ [24]. The model applied on the $200 \mathrm{GeV}$ data used a complete chemical equilibrium until thermal freeze-out [25]. With this model, it was therefore not possible to reproduce both spectra and elliptic flow. The Kolb et al. model can approximately reproduce the spectra but the default value for the elliptic flow is too large $[26]$.

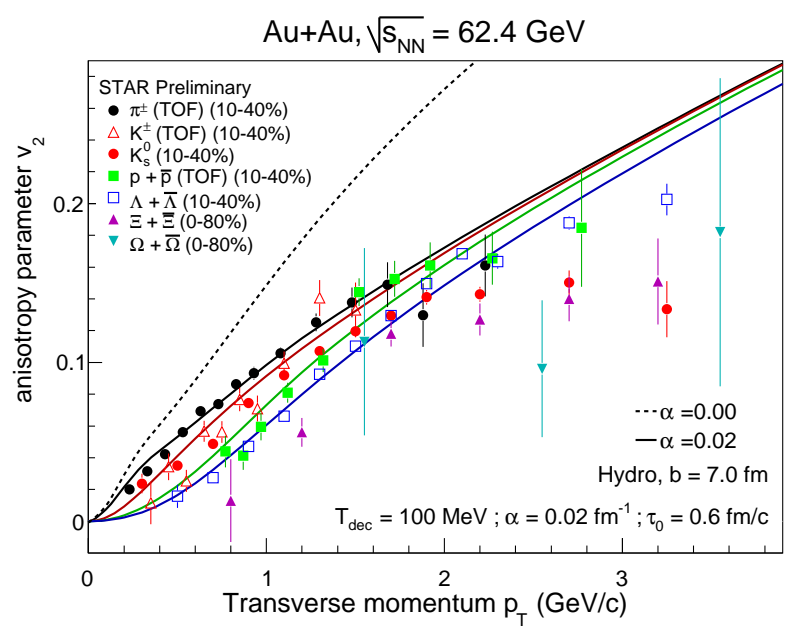

Fig. 8. Elliptic flow with respect to transverse momentum for identified particles in minbias (0-80\%) $\mathrm{Au}+\mathrm{Au}$ collisions. The data (points) are superposed with hydrodynamical calculations using $[22,23]$. The dotted line corresponds to the default ( $\alpha=0.00 \mathrm{fm}^{-1}$; for $\pi^{-}$only) and the full lines are obtained with $\alpha=0.02 \mathrm{fm}^{-1}$. The full lines are, from top to bottom, for $\pi^{-}, K^{-}, p$ and $\Lambda$.

In Fig.8, the elliptic flow as a function of transverse momentum is represented for different identified particles in $\mathrm{Au}+\mathrm{Au}$ collisions at $62.4 \mathrm{GeV}$. As at $200 \mathrm{GeV}$, the default value for $\alpha\left(0.00 \mathrm{fm}^{-1}\right)$ generates too much flow, so that the hydrodynamical calculations overshoot the data (dotted line). The use of $\alpha=0.02 \mathrm{fm}^{-1}$ (full lines) allows to simultaneously reproduce spectra and elliptic flow with the same parameters. This reproduction of elliptic flow consists, as at $200 \mathrm{GeV}$, of the mass ordering at low- $p_{T}$ and the qualitative agreement of data with hydrodynamical calculations until $p_{T} \sim 2 \mathrm{GeV} / \mathrm{c}$.

\section{Summary and Conclusion}

The high quality and quantity of data at different energies allow for systematic comparison of physics observables and excitation functions of strange and multi-strange particles. Thermal model fits lead to results at the intermediate energy of $62.4 \mathrm{GeV}$ that are almost identical to the results obtained at $200 \mathrm{GeV}$, in terms of chemical freezeout temperature and strangeness saturation factor. The strange and multi-strange anti-baryon to baryon $p_{T}$ integrated ratios expose a smooth evolution from SPS to top RHIC energies.

Dynamical properties are investigated using a BlastWave parameterization and an ideal hydrodynamical model description. The agreement of the hydrodynamical models with measured central spectra for light particles may be slighty worse at $62.4 \mathrm{GeV}$ than what was observed with the same model at $200 \mathrm{GeV}$. Within the framework of this model, the same thermal freeze-out temperature allows a reasonable description of all spectra at the lower and top RHIC energies, including multi-strange baryons. The apparent discrepency between hydrodynamical model calculations and Blast-Wave results may be investigated further with more precise measurements at lower $p_{T}$ for the multi-strange baryons $(<0.7 \mathrm{GeV} / \mathrm{c})$. The additional parameter $\alpha$ in the considered hydrodynamical model allows for a simultaneous description of spectra and elliptic flow with the use of an equation of state that involves a phase transition from a QGP to a hadron gas.

Although complete ideal hydrodynamical calculations do not reproduce all the data $\left(p_{T}>2 \mathrm{GeV} / \mathrm{c}\right.$, peripheral, high $\eta, \ldots)$ they give an indication as to what extent the system behaves thermally. In that matter they give the best ever observed agreement with data at RHIC energies. The $\alpha$ parameter in the Kolb et al. model that we used tries a basic description of the pre-equilibrium phase. This parameter as well as the use of a partial chemical equilibrium may to some extent already be considered as extentions to an ideal hydrodynamical description. The whole description may then be obtained with more sophisticated extensions as the combination of ideal hydrodynamics with microscopic cascade calculations $[27,28]$ or by introducing viscosity [29].

\section{References}

1. J. Rafelski and B. Müller, Phys. Rev. Lett. 48, (1982) 1066

2. K.H Ackermann et al., Nucl. Instrum. Meth. A 499, (2003) 624 
3. M. Anderson et al., Nucl. Instrum. Meth. A 499, (2003) 659

4. J. Adams et al. (STAR Collaboration), nucl-ex/0606014, submitted to Phys. Rev. Lett.

5. J. Takahashi (for the STAR Collaboration), J. Phys. G : Nucl. Part. Phys. 41 (2004) S1061

6. J. Speltz (for the STAR Collaboration), J. Phys. G : Nucl. Part. Phys. 41 (2004) S1025

7. P. Braun-Munzinger et al., Phys. Lett. B 518 (2001) 41

8. F. Becattini et al., Phys. Rev. C 69 (2004) 024905

9. J. Cleymans et al., Phys. Rev. C 65 (2004) 027901

10. J. Rafelski et al., Phys. Rev. C 72 (2005) 024905

11. M. Kaneta and N. Xu, nucl-ex/0405068

12. O. Barannikova (for the STAR Collaboration), nuclex/0403014

13. J. Adams et al. (STAR Collaboration), nucl-ex/0311017

14. F. Karsch et al., Eur. Phys. J. C 29 (2003) 549

15. J. Cleymans et al., Phys. Rev. C 73 (2006) 034905

16. H. Satz, Nucl. Phys. A 715 (2003) 3; V. Koch, Nucl. Phys. A $\mathbf{7 1 5}$ (2003) 108

17. H. Caines et al., J. Phys. G 27 (2001) 311; F. Antinori et al., Phys. Lett. B 595 (2004) 68; C. Adler et al., Phys. Rev. Lett. 89 (2002) 092301; T. Anticic et al., Phys. Rev. Lett. 93 (2004) 022302; J. Adams et al., Phys. Rev. Lett. 92 (2004) 027901

18. J.-Y. Ollitrault, Phys. Rev. D 46 (1992) 229

19. F. Retiere and M. Lisa, Phys. Rev. C 70 (2004) 044907

20. E. Schnedermann et al., Phys. Rev. C 48 (1993) 2462

21. C. Alt et al., Phys. Rev. Lett 94 (2005) 192301

22. P.F. Kolb et al., Phys. Rev. C 62 (2000) 054909; P.F. Kolb and R. Rapp, Phys. Rev. C 67 (2003) 044903; P.F. Kolb and U. Heinz, nucl-th/0305084

23. P.F. Kolb, http://nt3.phys.columbia.edu/OSCAR/models 24. J. Adams et al. (STAR Collaboration), Phys. Rev. C 72 (2005) 014904

25. P. Huovinen et al., Phys. Lett. B 503 (2001) 58

26. K. Adcox et al. (PHENIX Collaboration), Nucl. Phys. A 757 (2005) 184

27. T. Hirano and M. Gyulassy, Nucl. Phys. A 769 (2006) 71

28. C. Nonaka and S.A. Bass, nucl-th/0607018

29. D. Teaney, Phys. Rev. C 68 (2003) 034913 